\title{
Chemical and antioxidative assessment of dietary turnip (Brassica rapa var. rapa L.)
}

\author{
Fátima Fernandes ${ }^{\mathrm{a}}$, Patrícia Valentão ${ }^{\mathrm{b}}$, Carla Sousa ${ }^{\mathrm{b}}$, José A. Pereira ${ }^{\mathrm{a}}$, \\ Rosa M. Seabra ${ }^{b}$, Paula B. Andrade ${ }^{b, *}$ \\ ${ }^{a}$ CIMO/Escola Superior Agrária, Instituto Politécnico de Bragança, Campus de Sta Apolónia, Apartado 1172, 5301-855 Bragança, Portugal \\ ${ }^{\mathrm{b}}$ REQUIMTE/Serviço de Farmacognosia, Faculdade de Farmácia Universidade do Porto, R. Aníbal Cunha, 164, 4050-047 Porto, Portugal
}

Received 18 September 2006; received in revised form 15 February 2007; accepted 25 April 2007

\begin{abstract}
The phenolic compounds and organic acids of turnip (Brassica rapa var. rapa L.) edible parts (leaves and stems, flower buds and roots) were determined by HPLC-DAD and HPLC-UV, respectively. The results revealed a profile composed of 14 phenolics (3- $p$-coumaroylquinic, caffeic, ferulic and sinapic acids, kaempferol 3- $O$-sophoroside-7- $O$-glucoside, kaempferol 3- $O$-sophoroside-7- $O$-sophoroside, kaempferol 3-O-(feruloyl/caffeoyl)-sophoroside-7- $O$-glucoside, kaempferol 3,7- $O$-diglucoside, isorhamnetin 3,7- $O$-diglucoside, kaempferol 3- $O$-sophoroside, 1,2-disinapoylgentiobiose, 1,2'-disinapoyl-2-feruloylgentiobiose, kaempferol 3-O-glucoside and isorhamnetin 3-O-glucoside) and six organic acids (aconitic, citric, ketoglutaric, malic, shikimic and fumaric acids). The quantification of the identified compounds showed kaempferol 3-O-sophoroside-7-O-glucoside, kaempferol 3-O-(feruloyl/caffeoyl)-sophoroside-7-O-glucoside, isorhamnetin 3,7-O-diglucoside and isorhamnetin 3-O-glucoside as the main phenolics, and malic acid as the organic acid present in highest amounts. A screening of the antioxidative potential was also performed by means of the DPPH radical scavenging assay. Turnip flower buds exhibited the strongest antioxidant capacity.
\end{abstract}

(C) 2007 Elsevier Ltd. All rights reserved.

Keywords: Turnip; Brassica rapa var. rapa L.; Phenolic compounds; Organic acids; Antioxidant activity

\section{Introduction}

The Brassicaceae family includes a wide range of horticultural crops, some of them with economic significance, extensively used in the diet throughout the world (Sasaki \& Takahashi, 2002). Turnip (Brassica rapa var. rapa L.) is one of the oldest cultivated vegetables that has been used for human consumption since prehistoric times (Liang et al., 2006). This species is notably popular in Europe, particularly in its colder regions. It grows well in cold climates and can be stored several months after harvest. In the Iberian Peninsula it constitutes, together

\footnotetext{
* Corresponding author. Tel.: +351 222078935; fax: +351222003977.

E-mail address: pandrade@ff.up.pt (P.B. Andrade).
}

with other Brassica species, a unique supply of vegetables during the winter.

Turnip leaves are usually light green, thin and sparsely pubescent. A white-fleshed, large global or tapered root develops at the base of the leaf petioles. Branched flowering stems are also produced. The flowers are clustered at the top of the raceme and are usually raised above the terminal buds. In Portugal, turnip is usually used for its leaves and roots. Bolting occurs in late winter followed by the formation of flower buds, which are also consumed before opening and while still green (Rosa, 1997). Turnip edible parts are commonly consumed as a boiled vegetable, being used in the preparation of soups and stews, too. In addition, the flower buds are also eaten sauteed, with a mixture of hot olive oil and garlic, and with rice. 
This species has been previously studied for the phenylpropanoids (Liang et al., 2006), volatile constituents (Wallbank \& Wheatley, 1976) and allozymes (Persson, Fält, \& Von Bothmer, 2001) of the leaves, glucosinolates from the flower buds (Rosa, 1997), tuberization ability of the epicotyl (Nishijima, Sugii, Fukino, \& Mochizuki, 2005) and fatty acid composition of the seed oil (Velasco, Goffman, \& Becker, 1998).

It has been noticed that increasing the consumption of fruits and vegetables is a practical strategy for consumers to optimize their health and to reduce the risk of chronic diseases. In fact, plant-based foods contain significant amounts of bioactive non-nutritive compounds, which provide desirable health benefits beyond basic nutrition. This association is often attributed to the antioxidant phytochemicals, namely phenolic compounds and organic acids (Liu, 2003; Pulido, Bravo, \& Saura-Calixto, 2000; Silva et al., 2004). These compounds are also known to contribute to the organoleptic characteristics of fruits and vegetables (Vaughan \& Geissler, 1997) and have been widely applied for their quality control (Ferreres et al., 2005; Silva et al., 2005; Sousa et al., 2005).

The phenolic (Ferreres et al., 2005, 2006; Llorach, GilIzquierdo, Ferreres, \& Tomás-Barberán, 2003; Romani, Vignolini, Isolani, Ieri, \& Heimler, 2006; Sousa et al., 2005; Vallejo, Tomás-Barberán, \& Ferreres, 2004; Vrchovska et al., 2006) and organic acid (Ferreres et al., 2006; Sousa et al., 2005; Vrchovska et al., 2006) composition of Brassica vegetables or byproducts has been widely investigated. In what concerns B. rapa, only the flavonoids in var. sylvestris (Romani et al., 2006) were characterized. Despite being much appreciated and highly consumed, in B. rapa var. rapa only the production of hydroxycinnamic acids conjugated with malate in methyl jasmonate treated leaves was described (Liang et al., 2006) and nothing has been reported about its organic acids.

The purpose of this study was to characterize the phenolic compounds and organic acids profiles of the distinct turnip edible parts (leaves and stems, flower buds and roots) and to assess their antiradical activity. Phenolic compounds and organic acids were determined by HPLCDAD and HPLC-UV, respectively, and the antioxidant capacity was evaluated by the DPPH radical scavenging assay.

\section{Materials and methods}

\subsection{Standards and reagents}

Caffeic, p-coumaric, sinapic, oxalic, citric, ketoglutaric, malic, succinic, shikimic and fumaric acids were from Sigma (St. Louis, MO, USA) and ferulic acid, cis-aconitic acid, kaempferol 3-O-glucoside and isorhamnetin 3-O-glucoside from Extrasynthése (Genay, France). Methanol and formic acid were obtained from Merck (Darmstadt, Germany) and sulfuric acid from Pronalab (Lisbon, Portugal).
The water was treated in a Milli-Q water purification system (Millipore, Bedford, MA, USA). DPPH was purchased from Sigma Chemical Co. (St. Louis, USA).

\subsection{Plant material}

Turnip samples were sowed in Carrazeda de Ansiães (northern Portugal), in September 2005, and grown without any fertilization treatment. After harvesting, in February 2006, the plant material of five distinct individuals was immediately transferred to the laboratory where leaves and stems, flower buds and roots were separated. The samples were kept in a freezer at $-20^{\circ} \mathrm{C}$, prior to their lyophilisation in a Labconco 4.5 Freezone apparatus (Kansas City, MO, USA). Then the dried material was powdered.

\subsection{Preparation of extracts}

Aqueous extracts of leaves and stems, flower buds and roots from turnip were prepared by putting ca. $3.0 \mathrm{~g}$ of dried material in $300 \mathrm{ml}$ of boiling water. The mixture was boiled for $15 \mathrm{~min}$ and then filtered over a Büchner funnel. The extracts were then lyophilised, each one yielding ca. $1.5 \mathrm{~g}$ of dry matter. The lyophilised extracts were kept in an exsicator, in the dark. For phenolic compounds and organic acids analysis, they were redissolved in water and in sulfuric acid $0.01 \mathrm{~N}$, respectively.

\subsection{HPLC-UV analyses of organic acids}

Analyses of organic acids were carried out as previously reported (Ferreres et al., 2006), using an analytical HPLC unit (Gilson) in conjunction with a column heating device set at $30^{\circ} \mathrm{C}$, and an ion exclusion column, Nucleogel ${ }^{\circledR}$ Ion $300 \mathrm{OA}(300 \times 7.7 \mathrm{~mm})$. Briefly, elution was developed isocratically at a solvent flow rate of $0.2 \mathrm{ml} / \mathrm{min}$, with sulfuric acid $0.01 \mathrm{~N}$. The detection was performed with an UV detector, at $214 \mathrm{~nm}$. Organic acids quantification was achieved by the absorbance recorded in the chromatograms relative to external standards, and the peaks in the chromatograms were integrated using a default baseline construction technique.

\subsection{HPLC-DAD analyses of phenolic compounds}

Analysis of phenolic compounds was carried out using an analytical HPLC unit (Gilson), equipped with a Gilson diode array detector. The compounds were separated by using a Spherisorb ODS2 $(25.0 \times 0.46 \mathrm{~cm} ; 5 \mu \mathrm{m}$, particle size) column and a solvent mixture of water (adjusted to $\mathrm{pH} 3.2$ with formic acid at 10\%, v/v) (A) and methanol (B). Elution was carried out at $1 \mathrm{ml} / \mathrm{min}$ and the solvent gradient system was as follows: $20 \% \mathrm{~B}$ at $0 \mathrm{~min}, 50 \% \mathrm{~B}$ at $35 \mathrm{~min}, 80 \% \mathrm{~B}$ at $45 \mathrm{~min}$ and $100 \% \mathrm{~B}$ at $50 \mathrm{~min}$. Spectral data from all peaks were accumulated in the $200-400 \mathrm{~nm}$ range, and chromatograms were acquired at $330 \mathrm{~nm}$. Data 
were processed by Unipoint system software (Gilson Medical Electronics, Villiers le Bel, France) and peak purity was checked by means of the software contrast facilities.

Phenolic compounds quantification was achieved by the absorbance recorded in the chromatograms relative to external standards. Since standards of several compounds identified in turnip were not commercially available, 3- $p$ coumaroylquinic acid was quantified as $p$-coumaric acid and kaempferol and isorhamnetin derivatives as kaempferol 3-O-glucoside and isorhamnetin 3-O-glucoside, respectively.

\subsection{DPPH radical scavenging activity}

The antiradical capacity of the distinct turnip edible parts was estimated spectrophotometrically in an ELX808 IU Ultra Microplate Reader (Bio-Tek Instruments, Inc.), by monitoring the disappearance of $\mathrm{DPPH}^{-}$ at $515 \mathrm{~nm}$, according to a described procedure (Ferreres et al., 2006). The reaction mixtures in the sample wells consisted of $25 \mu \mathrm{l}$ aqueous extract (five different concentrations) and $200 \mu \mathrm{l} \mathrm{DPPH}^{-}$dissolved in methanol. The mixture was left to stand for $30 \mathrm{~min}$ at room temperature. Three experiments were performed in triplicate.

The scavenging activity (SA) was calculated as a percentage of $\mathrm{DPPH}^{-}$discolouration using the equation: $\% \mathrm{SA}=100 *(1-\mathrm{Ac} / \mathrm{Ad})$, where Ac is the absorbance of the resulting solution when the extract has been added at a particular concentration, and Ad is the absorbance of the DPPH solution. The extract concentration providing $25 \%$ inhibition $\left(\mathrm{IC}_{25}\right)$ was calculated from the graph of SA percentage against extract concentration.

\subsection{Statistical analysis}

The evaluation of statistical significance was determined by ANOVA, followed by Newman-Keuls test. The level of significance was set at $p<0.05$.

\section{Results and discussion}

\subsection{Phenolic compounds in turnip}

The HPLC-DAD analysis of turnip aqueous extracts revealed the presence of several hydroxycinnamic and flavonoid derivatives. By this means in turnip flower buds (Fig. 1a) and leaves and stems (Fig. 1b), it was possible to identify 14 phenolic compounds: 3-p-coumaroylquinic, caffeic, ferulic and sinapic acids, kaempferol 3-O-sophoroside-7-O-glucoside, kaempferol 3-O-sophoroside-7- $O$-sophoroside, kaempferol 3-O-(feruloyl/caffeoyl)-sophoroside7-O-glucoside, kaempferol 3,7-O-diglucoside, isorhamnetin 3,7-O-diglucoside, kaempferol 3-O-sophoroside, 1,2-disinapoylgentiobiose, 1,2'-disinapoyl-2-feruloylgentiobiose, kaempferol 3-O-glucoside and isorhamnetin 3-O-glucoside (Fig. 2). Caffeic, ferulic and sinapic acids, kaempferol 3- $O$ -
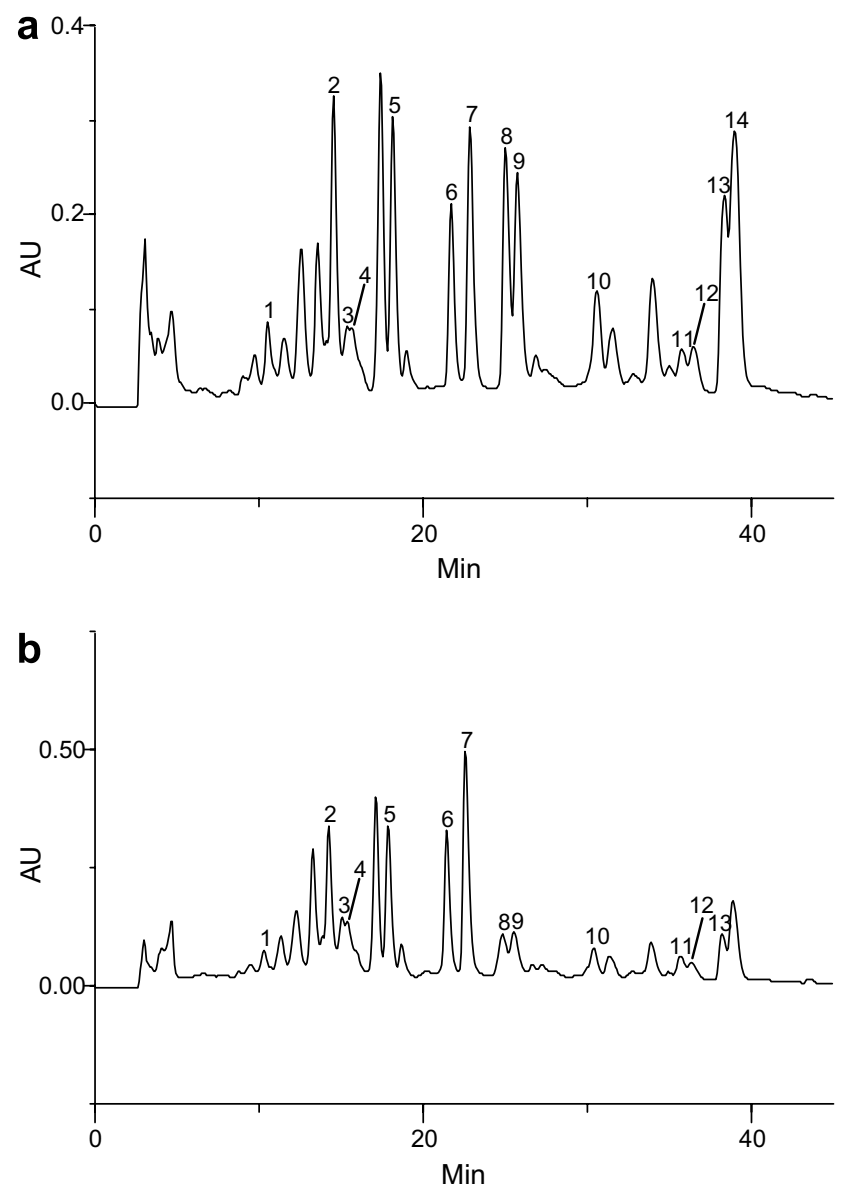

Fig. 1. HPLC-DAD of phenolic compounds in turnip (a) flower buds and (b) leaves and stems. Detection at $330 \mathrm{~nm}$. Peaks: (1) 3-p-coumaroylquinic acid; (2) kaempferol 3-O-sophoroside-7-O-glucoside; (3) kaempferol 3-Osophoroside-7- $O$-sophoroside (4) caffeic acid; (5) kaempferol 3- $O$-(feruloyl/ caffeoyl)-sophoroside-7- $O$-glucoside; (6) kaempferol 3,7-O-diglucoside; (7) isorhamnetin 3,7-O-diglucoside; (8) ferulic acid; (9) sinapic acid; (10) kaempferol 3-O-sophoroside; (11) 1,2-disinapoylgentiobiose; (12) 1,2'disinapoyl-2-feruloylgentiobiose; (13) kaempferol 3-O-glucoside; (14) isorhamnetin 3-O-glucoside.

glucoside and isorhamnetin 3-O-glucoside were identified by comparison of their retention times and UV-visible spectra in the range of $200-400 \mathrm{~nm}$, with those of authentic standards. Kaempferol 3-O-sophoroside-7-O-glucoside, kaempferol 3-O-sophoroside-7-O-sophoroside, kaempferol 3-O-(feruloyl/caffeoyl)-sophoroside-7-O-glucoside, kaempferol 3-O-sophoroside, 3-p-coumaroylquinic acid, 1,2-disinapoylgentiobiose and 1,2'disinapoyl-2-feruloylgentiobiose were identified according to our previous works in $B$. oleracea L. var costata DC (Ferreres et al., 2005, 2006). Using the same described experimental conditions, similar chromatograms were obtained for these compounds, in which they had the same order of elution, the same retention time and the same UV-visible spectra. Kaempferol 3,7-O-diglucoside and isorhamnetin 3,7-O-diglucoside were identified according to their chromatographic behaviour and characteristic UV-visible spectra, as described in $B$. rapa subsp. sylvestris by Romani et al. (2006); kaempferol 
<smiles>O=C(/C=C/c1ccc(O)cc1)OC1CC(O)(C(=O)O)CC(O)C1O</smiles><smiles>[R6]Oc1cc(O)c2c(=O)c(O[R20])c(-c3ccc(O)c([R])c3)oc2c1</smiles>

$\begin{array}{llll} & \mathbf{R}_{1} & \mathbf{R}_{2} & \mathbf{R}_{\mathbf{3}} \\ \mathbf{2} & \mathrm{H} & \text { sophorose } & \text { glucose } \\ \mathbf{3} & \mathrm{H} & \text { sophorose } & \text { sophorose } \\ \mathbf{5} & \mathrm{H} & \text { (feruloyl/caffeoyl)-sophorose } & \text { glucose } \\ \mathbf{6} & \mathrm{H} & \text { glucose } & \text { glucose } \\ \mathbf{7} & \mathrm{OCH}_{3} & \text { glucose } & \text { glucose } \\ \mathbf{1 0} & \mathrm{H} & \text { sophorose } & \mathrm{H} \\ \mathbf{1 3} & \mathrm{H} & \text { glucose } & \mathrm{H} \\ \mathbf{1 4} & \mathrm{OCH}_{3} & \text { glucose } & \mathrm{H}\end{array}$<smiles>[R]C/C=C/c1cc([R])c(O)c([R])c1</smiles>

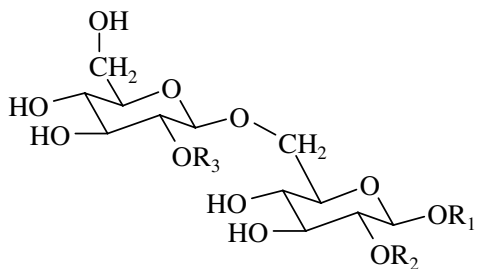<smiles>CO[Ga]O[Mg]</smiles>

$\begin{array}{llll} & \mathbf{R}_{\mathbf{1}} & \mathbf{R}_{\mathbf{2}} & \mathbf{R}_{\mathbf{3}} \\ \mathbf{1 1} & \text { sinapoyl } & \text { sinapoyl } & \mathrm{H} \\ \mathbf{1 2} & \text { sinapoyl } & \text { feruloyl } & \text { sinapoyl }\end{array}$

Fig. 2. Chemical structures of the identified turnip phenolics: (1) 3-p-coumaroylquinic acid; (2) kaempferol 3-O-sophoroside-7-O-glucoside; (3) kaempferol 3-O-sophoroside-7- $O$-sophoroside (4) caffeic acid; (5) kaempferol 3-O-(feruloyl/caffeoyl)-sophoroside-7-O-glucoside; (6) kaempferol 3,7-Odiglucoside; (7) isorhamnetin 3,7-O-diglucoside; (8) ferulic acid; (9) sinapic acid; (10) kaempferol 3-O-sophoroside; (11) 1,2-disinapoylgentiobiose; (12) 1,2'-disinapoyl-2-feruloylgentiobiose; (13) kaempferol 3-O-glucoside; (14) isorhamnetin 3-O-glucoside.

Table 1

Phenolic compounds in turnip samples $\left(\mathrm{mg} / \mathrm{kg}\right.$, dry basis) ${ }^{\mathrm{a}}$

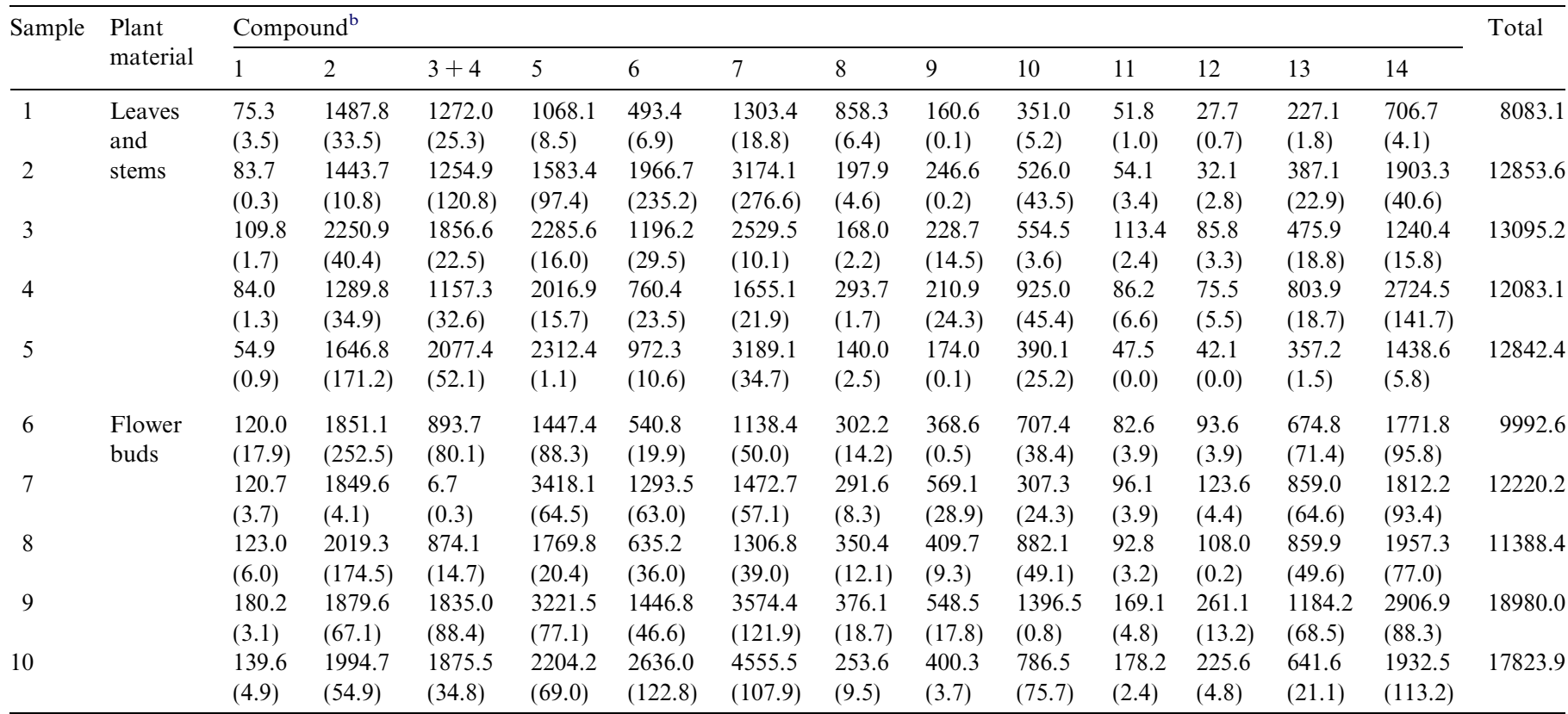

\footnotetext{
${ }^{\text {a }}$ Results are expressed as mean (standard deviation) of three determinations.

b (1) 3-p-Coumaroylquinic acid; (2) kaempferol 3-O-sophoroside-7- $O$-glucoside; (3) kaempferol 3-O-sophoroside-7-O-sophoroside (4) caffeic acid; (5) kaempferol 3-O-(feruloyl/caffeoyl)-sophoroside-7- $O$-glucoside; (6) kaempferol 3,7-O-diglucoside; (7) isorhamnetin 3,7- $O$-diglucoside; (8) ferulic acid; (9) sinapic acid; (10) kaempferol 3-O-sophoroside; (11) 1,2-disinapoylgentiobiose; (12) 1,2'-disinapoyl-2-feruloylgentiobiose; (13) kaempferol 3-O-glucoside; (14) isorhamnetin 3-O-glucoside.
} 
3,7-O-diglucoside exhibits two maxima at 266 and $349 \mathrm{~nm}$ and a shoulder at $318 \mathrm{~nm}$, while isorhamnetin 3,7-O-diglucoside shows its maxima at 255 and $354 \mathrm{~nm}$ and a shoulder at $268 \mathrm{~nm}$. Turnip roots showed a distinct composition, in which only ferulic and sinapic acids and their derivatives were detected in vestigial amounts. No qualitative differences were observed among the five samples of each plant material (Table 1). As far as we know, all these compounds are reported for the first time in turnip edible parts. Comparing the results obtained with those described before for another $B$. rapa variety, namely var. sylvestris (Romani et al., 2006), it can be seen that kaempferol 3,7-O-diglucoside, isorhamnetin 3,7-O-diglucoside, kaempferol 3-O-sophoroside, 1,2-disinapoylgentiobiose, 1,2'-disinapoyl-2-feruloylgentiobiose, kaempferol 3-O-glucoside and isorhamnetin 3-O-glucoside are the only common compounds.

The quantification of the phenolics present in the different turnip aqueous extracts revealed a high amount of these
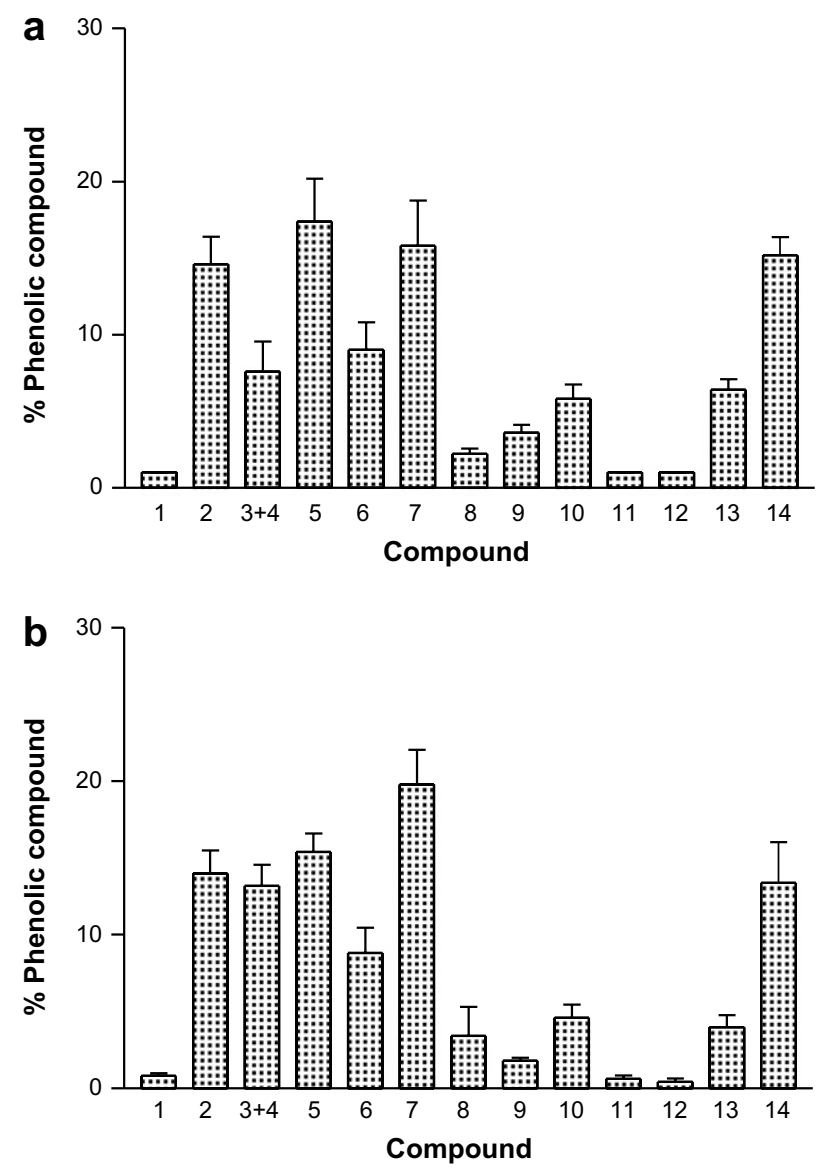

Fig. 3. Phenolic profile of turnip (a) flower buds and (b) leaves and stems. Values represent mean, and standard error bars are on the top of each column. (1) 3-p-Coumaroylquinic acid; (2) kaempferol 3- $O$-sophoroside-7$O$-glucoside; (3) kaempferol 3-O-sophoroside-7- $O$-sophoroside (4) caffeic acid; (5) kaempferol 3-O-(feruloyl/caffeoyl)-sophoroside-7- $O$-glucoside; (6) kaempferol 3,7-O-diglucoside; (7) isorhamnetin 3,7-O-diglucoside; (8) ferulic acid; (9) sinapic acid; (10) kaempferol 3-O-sophoroside; (11) 1,2disinapoylgentiobiose; (12) 1,2'-disinapoyl-2-feruloylgentiobiose; (13) kaempferol 3-O-glucoside; (14) isorhamnetin 3-O-glucoside. compounds in the flower buds (ranging from 10 to $19 \mathrm{~g} / \mathrm{kg}$, dry basis) and in the leaves and stems (varying from 8 to $13 \mathrm{~g} / \mathrm{kg}$, dry basis), in which flavonols were always the major compounds (Table 1).

In turnip flower buds, kaempferol 3- $O$-sophoroside-7-Oglucoside, kaempferol 3- $O$-(feruloyl/caffeoyl)-sophoroside7-O-glucoside, isorhamnetin 3,7-O-diglucoside and isorhamnetin 3-O-glucoside were the major compounds, each one corresponding to ca. $15-17 \%$ of total phenolics. 3-p-Coumaroylquinic acid, 1,2-disinapoylgentiobiose and $1,2^{\prime}$-disinapoyl-2-feruloylgentiobiose were the compounds present in minor amounts, each one representing $1 \%$ of total phenolics (Table 1, Fig. 3a). The leaves and stems exhibited a similar profile, in which kaempferol 3- $O$-sophoroside-7-Oglucoside, kaempferol 3-O-(feruloyl/caffeoyl)-sophoroside7-O-glucoside, isorhamnetin 3,7-O-diglucoside and isorhamnetin 3-O-glucoside were also the compounds in higher contents (each one varying from ca. $13 \%$ to $20 \%$ of total phenolics) and 3-p-coumaroylquinic acid, 1,2-disinapoylgentiobiose and 1,2'-disinapoyl-2-feruloylgentiobiose the minor ones (corresponding each to less than ca. 0.8\%) (Table 1, Fig. 3b). However, turnip flower buds showed significantly lower amounts of the pair kaempferol 3-O-sophoroside-7-O-sophoroside plus caffeic acid and presented significantly higher contents of sinapic acid, 1,2'-disinapoyl-2-feruloylgentiobiose and kaempferol 3-O-glucoside than those from the leaves and stems.

\subsection{Organic acids in turnip}

The three analysed turnip edible parts showed similar organic acid composition (Fig. 4). Generally, aconitic, citric, ketoglutaric, malic, shikimic and fumaric acids were detected in all parts, but some qualitative differences were noticed within samples of the same plant material (Table 2). Shikimic acid was not present in leaves and stems sample 5, neither in flower buds sample 10 nor in roots samples 11 and 15. These results suggest the occurrence of some

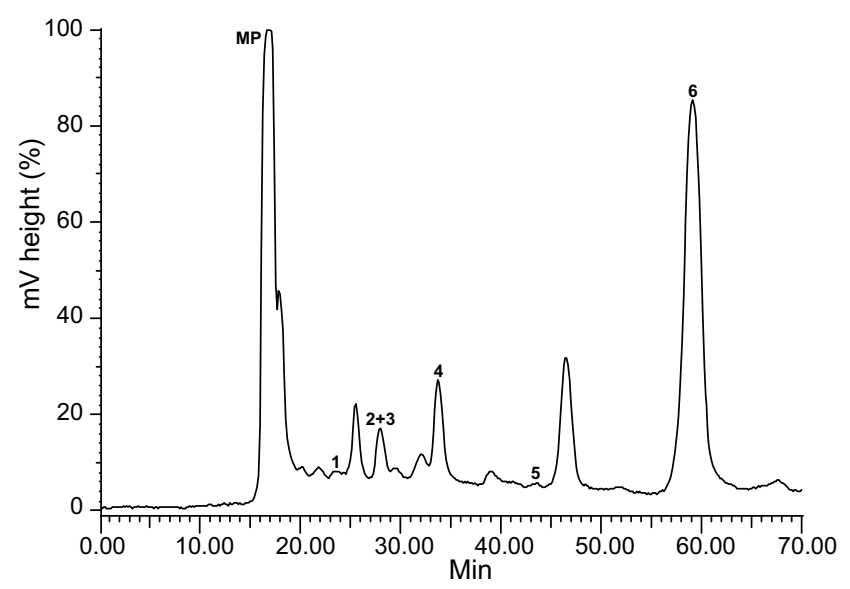

Fig. 4. HPLC-UV of organic acids in turnip leaves and stems. Detection at $214 \mathrm{~nm}$. Peaks: (MP) mobile phase; (1) aconitic acid; (2) citric acid; (3) ketoglutaric acid; (4) malic acid; (5) shikimic acid; (6) fumaric acid. 
Table 2

Organic acids in turnip samples $(\mathrm{mg} / \mathrm{kg} \text {, dry basis })^{\mathrm{a}}$

\begin{tabular}{|c|c|c|c|c|c|c|c|}
\hline \multirow[t]{2}{*}{ Sample } & \multirow[t]{2}{*}{ Plant material } & \multicolumn{5}{|l|}{ Compound $^{\mathrm{b}}$} & \multirow[t]{2}{*}{ Total } \\
\hline & & 1 & $2+3$ & 4 & 5 & 6 & \\
\hline 1 & \multirow[t]{5}{*}{ Leaves and stems } & $160.8(3.6)$ & $18565.1(2100.5)$ & $34403.4(1059.7)$ & $303.3(7.9)$ & $542.6(19.5)$ & 53975.2 \\
\hline 2 & & $3030.7(804.3)$ & $5241.2(435.6)$ & $18800.0(981.0)$ & $19.2(0.0)$ & $835.2(0.2)$ & 27926.2 \\
\hline 3 & & $180.2(2.0)$ & $14002.8(47.6)$ & $34954.8(1417.1)$ & $296.1(47.6)$ & $391.3(0.2)$ & 49825.1 \\
\hline 4 & & $12472.0(228.1)$ & $18437.0(432.7)$ & $39296.8(10.5)$ & $228.6(32.2)$ & $1683.5(22.5)$ & 72117.9 \\
\hline 5 & & $251.7(9.8)$ & $15458.4(72.1)$ & $34898.6(667.3)$ & - & $873.0(9.9)$ & 51481.8 \\
\hline 6 & \multirow[t]{5}{*}{ Flower buds } & $5251.0(137.3)$ & $13871.1(4535.2)$ & $20727.5(90.4)$ & $49.4(0.8)$ & $1502.2(18.4)$ & 41401.2 \\
\hline 7 & & $3065.8(178.2)$ & $4346.4(224.2)$ & $8574.8(268.8)$ & $126.2(25.8)$ & $959.7(14.4)$ & 17072.9 \\
\hline 8 & & $5244.5(250.7)$ & $10996.5(829.6)$ & $15071.9(61.1)$ & $80.9(9.1)$ & $1132.3(12.1)$ & 32526.2 \\
\hline 9 & & $18912.3(1222.7)$ & $34083.2(2855.0)$ & $27258.5(6551.3)$ & $3.9(1.1)$ & $1610.1(0.5)$ & 81868.0 \\
\hline 10 & & $565.5(3.6)$ & 34936.8 (1988.4) & $25886.8(694.3)$ & - & $954.2(0.9)$ & 62343.4 \\
\hline 11 & \multirow[t]{5}{*}{ Roots } & $95.1(0.7)$ & $4978.8(114.1)$ & $28814.2(145.2)$ & - & $891.4(0.7)$ & 34779.5 \\
\hline 12 & & $103.6(7.4)$ & $5856.1(100.4)$ & $32637.8(60.7)$ & $16.2(0.5)$ & $1325.7(16.5)$ & 39939.4 \\
\hline \multirow[t]{2}{*}{13} & & $52.3(4.5)$ & $2972.1(36.3)$ & $18868.4(100.3)$ & $146.2(7.4)$ & $537.0(55.1)$ & 22576.0 \\
\hline & & $2819.5(61.4)$ & $5965.8(124.9)$ & $30611.2(385.0)$ & $33.3(0.1)$ & $1320.0(15.0)$ & 40749.8 \\
\hline 15 & & $139.8(4.4)$ & $6225.4(403.9)$ & $35340.8(660.3)$ & - & $650.6(7.1)$ & 42356.6 \\
\hline
\end{tabular}

${ }^{\mathrm{a}}$ Results are expressed as mean (standard deviation) of three determinations.

b (1) Aconitic acid; (2) citric acid; (3) ketoglutaric acid; (4) malic acid; (5) shikimic acid; (6) fumaric acid.

variability in the organic acids composition of turnip edible parts. All these compounds are described for the first time in turnip.

In a general way, the quantification of the identified organic acids showed a high organic acid content (ranging from 36 to $51 \mathrm{~g} / \mathrm{kg}$, dry basis), with a tendency for higher content of these compounds in flower buds and in leaves and stems than in the roots (Table 2). Three distinct organic acid profiles were obtained (Fig. 5). When present, shikimic acid was the minor compound, representing from ca. $0.1 \%$ to $0.3 \%$ of total acids (Fig. 5), with the exceptions of leaves and stems samples 1 and 3 and root sample 13, in which aconitic acid was the compound present in lower amounts (Table 2).

Malic acid was the major compound in the three distinct turnip edible parts (Fig. 5), except for flower buds samples 9 and 10, which presented the pair citric plus ketoglutaric acids in the highest content (Table 2). In fact, when comparing malic acid content in the three analysed parts, it was revealed to be significantly distinct for all of them: roots exhibited a significantly higher amount (ca. 81\%), followed by leaves and stems (ca. 65\%) and flower buds showed a significantly lower content (ca. 44\%) (Fig. 5). Thus, malic acid content may be useful to distinguish turnip edible parts.

In addition, flower buds showed the highest aconitic acid relative amount (ca. 14\%) (Fig. 5a), which was significantly higher than that found in roots (ca. 2\%) (Fig. 5c). The pair citric plus ketoglutaric acids also revealed to be significantly lower in turnip roots (Fig. 5c).

\subsection{Antioxidant activity}

The DPPH* assay is considered a valid and easy assay to evaluate radical scavenging activity of antioxidants, since the radical compound is stable and does not have to be generated as in other radical scavenging assays. When it reacts with hydrogen donors, the DPPH radical is reduced to the corresponding hydrazine; a decrease in absorbance at $515 \mathrm{~nm}$ is produced by the addition of the antioxidant (Fukumoto \& Mazza, 2000).

Turnip edible parts displayed a concentration-dependent scavenging activity (Fig. 6). $\mathrm{IC}_{25}$ values were determined in order to compare the results, once it was not possible to reach $50 \%$ scavenging activity with all samples and considering that it corresponds approximately to the middle activity of each curve. The flower buds were revealed to be the most active part (mean $\mathrm{IC}_{25}$ of $0.47 \mathrm{mg} / \mathrm{ml}$ ), followed by the leaves and stems (mean $\mathrm{IC}_{25}$ at $0.56 \mathrm{mg} / \mathrm{ml}$ ). Turnip roots showed a significantly lower antioxidant capacity, with a mean $\mathrm{IC}_{25}$ of $1.44 \mathrm{mg} /$ $\mathrm{ml}$.

When comparing these results with those we obtained before in the same assay for B. oleracea var costata, it could be noticed that turnip flower buds exhibits similar antioxidant capacity as both $B$. oleracea var costata external and internal leaves (Ferreres et al., 2006; Vrchovska et al., 2006), but it is much weaker than that of its seeds (Ferreres et al., 2007).

The antioxidant potential exhibited by the different turnip edible parts is obviously determined by their composition. The $\mathrm{IC}_{25}$ values found in the DPPH assay are correlated with both total phenolics and organic acids amounts. However, despite the observation that the three parts tested did not exhibit significantly different total organic acids content, the roots displayed a significantly smaller antioxidant capacity. Thus, it seems that phenolics represent the main contribution for the resulting effect, and leaves and stems and flower buds showed the highest antioxidant potential. In addition, hydroxycinnamic acids and 

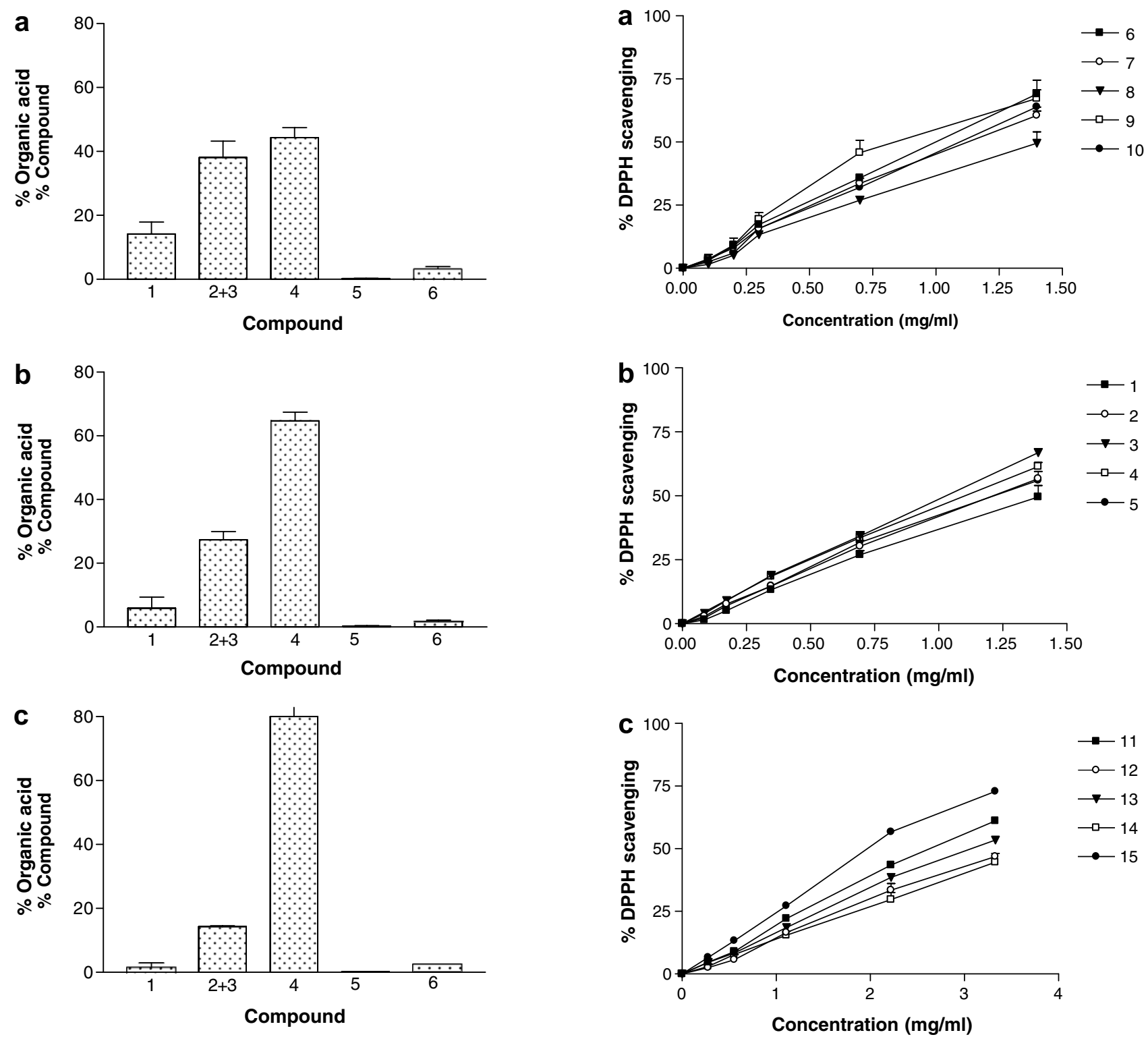

Fig. 5. Organic acids profile of turnip: (a) flower buds, (b) leaves and stems and (c) roots. Values represent mean, and standard error bars are on the top of each column. (1) aconitic acid; (2) citric acid; (3) ketoglutaric acid; (4) malic acid; (5) shikimic acid; (6) fumaric acid.

their derivatives (Fukumoto \& Mazza, 2000; Plumb, Price, Rhodes, \& Williamson, 1997), flavonol glycosides (Braca et al., 2003; Tang, Lou, Wang, Li, \& Zhuang, 2001) or organic acids (Silva et al., 2004) are known to exert antioxidant activity.

In conclusion, the results obtained in this work are very promising, indicating that turnip may be an easily accessible dietary source of biologically active compounds. However, its root seems to be the less interesting edible part, due to the very low amount of phenolic compounds and small antioxidant capacity. The phenolics and organic acids profiles of turnip samples from other geographic origins should be determined, in order to confirm whether these metabolites may be useful chemical markers of this vegetable.

Fig. 6. Effect of turnip edible parts on DPPH reduction: (a) flower buds; (b) leaves and stems; (c) roots; (1-15) samples. Values show mean $\pm \mathrm{SE}$ from 3 experiments performed in triplicate.

\section{Acknowledgements}

F. Fernandes is indebted to "Programa Ciência e Inovação 2010, Medida IV.7" for the grant. The authors are grateful to Fundação para a Ciência e a Tecnologia for financial support of this work (PTDC/AGR-AAM/ 64150/2006).

\section{References}

Braca, A., Fico, G., Morelli, I., De Simone, F., Tomè, F., \& De Tommasi, N. (2003). Antioxidant and free radical scavenging activity of flavonol glycosides from different Aconitum species. Journal of Ethnopharmacology, 86, 63-67.

Ferreres, F., Sousa, C., Valentão, P., Seabra, R. M., Pereira, J. A., \& Andrade, P. B. (2007). Tronchuda cabbage (Brassica oleraceae L. var. 
costata DC) seeds: Phytochemical characterization and antioxidant potential. Food Chemistry, 101, 549-558.

Ferreres, F., Sousa, C., Vrchovska, V., Valentão, P., Pereira, J. A., Seabra, R. M., et al. (2006). Chemical composition and antioxidant activity of tronchuda cabbage internal leaves. European Food Research and Technology, 222, 88-98.

Ferreres, F., Valentão, P., Llorach, R., Pinheiro, C., Cardoso, L., Pereira, J. A., et al. (2005). Phenolic compounds in external leaves of tronchuda cabbage (Brassica oleracea L. var. costata DC). Journal of Agricultural and Food Chemistry, 53, 2901-2907.

Fukumoto, L. R., \& Mazza, G. (2000). Assessing antioxidant and prooxidant activities of phenolic compounds. Journal of Agricultural and Food Chemistry, 48, 3597-3604.

Liang, Y.-S., Kim, H. K., Lefeber, A. W. M., Erkelens, C., Choi, Y. H., \& Verpoorte, R. (2006). Identificaion of phenylpropanoids in methyl jasmonate treated Brassica rapa leaves using two-dimensional nuclear magnetic resonance spectroscopy. Journal of Chromatography A, 1112, $148-155$.

Liu, R. H. (2003). Health benefits of fruit and vegetables are from additive and synergistic combinations of phytochemicals. American Journal of Clinical Nutrition, 78(Suppl), 517S-520S.

Llorach, R., Gil-Izquierdo, A., Ferreres, F., \& Tomás-Barberán, F. A. (2003). HPLC-DAD-MS/MS ESI characterization of unusual highly glycosylated acylated flavonoids from cauliflower (Brassica oleracea L. var. botrytis) agroindustrial byproducts. Journal of Agricultural and Food Chemistry, 51, 3895-3899.

Nishijima, T., Sugii, H., Fukino, N., \& Mochizuki, T. (2005). Aerial tubers induced in turnip (Brassica rapa L. var. rapa (L.) Hartm.) by gibberellin treatment. Scientia Horticulturae, 105, 423-433.

Persson, K., Fält, A.-S., \& Von Bothmer, R. (2001). Genetic diversity of allozymes in turnip (Brassica rapa L. var. rapa) from the Nordic area. Hereditas, 134, 43-52.

Plumb, G. W., Price, K. R., Rhodes, M. J., \& Williamson, G. (1997). Antioxidant properties of the major polyphenolic compounds in broccoli. Free Radical Research, 27, 429-435.

Pulido, R., Bravo, L., \& Saura-Calixto, F. (2000). Antioxidant activity of dietary polyphenols as determined by a modified ferric reducing/ antioxidant power assay. Journal of Agricultural and Food Chemistry, 48, 3396-3402.

Romani, A., Vignolini, P., Isolani, L., Ieri, F., \& Heimler, D. (2006). HPLC-DAD/MS characterization of flavonoids and hydroxycinnamic derivatives in turnip tops (Brassica rapa L. Subsp. sylvestris L.). Journal of Agricultural and Food Chemistry, 54, 1342-1346.

Rosa, E. A. S. (1997). Glucosinolates from flower buds of Portuguese Brassica crops. Phytochemistry, 44, 1415-1419.

Sasaki, K., \& Takahashi, T. (2002). A flavonoid from Brassica rapa flower as the UV-absorbing nectar guide. Phytochemistry, 61, 339-343.

Silva, B. M., Andrade, P. B., Martins, R. C., Valentão, P., Ferreres, F., Seabra, R. M., et al. (2005). Quince (Cydonia oblonga Miller) fruit characterization using principal component analysis. Journal of Agricultural and Food Chemistry, 53, 111-122.

Silva, B. M., Andrade, P. B., Valentão, P., Ferreres, F., Seabra, R. M., \& Ferreira, M. A. (2004). Quince (Cydonia oblonga Miller) fruit (pulp, peel and seed) and jam: Antioxidant activity. Journal of Agricultural and Food Chemistry, 52, 4705-4712.

Sousa, C., Valentão, P., Rangel, J., Lopes, G., Pereira, J. A., Ferreres, F., et al. (2005). Influence of two fertilization regimens on the amounts of organic acids and phenolic compounds of tronchuda cabbage (Brassica oleracea L. var. costata DC). Journal of Agricultural and Food Chemistry, 53, 9128-9132.

Tang, Y., Lou, F., Wang, J., Li, Y., \& Zhuang, S. (2001). Coumaroyl flavonol glycosides from the leaves of Ginkgo biloba. Phytochemistry, $58,1251-1256$.

Vallejo, F., Tomás-Barberán, F. A., \& Ferreres, F. (2004). Characterisation of flavonols in broccoli (Brassica oleracea L. var. italica) by liquid chromatography-UV diode-array detection-electrospray ionisation mass spectrometry. Journal of Chromatography A, 1054, 181-193.

Vaughan, J. G., \& Geissler, C. A. (1997). The new oxford book of food plants. New York: Oxford University Press, pp. 196.

Velasco, L., Goffman, F. D., \& Becker, H. C. (1998). Variability for the fatty acid composition of the seed oil in germplasm collection of the genus Brassica. Genetic Resources Crop Evolution, 45, 371-382.

Vrchovska, V., Sousa, C., Valentão, P., Ferreres, F., Pereira, J. A., Seabra, R. M., et al. (2006). Antioxidative properties of tronchuda cabbage (Brassica oleracea L. var. costata DC) external leaves against DPPH, superoxide radical, hydroxyl radical and hypochlorous acid. Food Chemistry, 98, 416-425.

Wallbank, B. E., \& Wheatley, G. A. (1976). Volatile constituents from cauliflower and other crucifers. Phytochemistry, 15, 763-766. 